\title{
Structures and Properties of Alumina-Based Ceramic for Reconstructive Oncology
}

\author{
M. V. Grigoriev ${ }^{1,2, a)}$ and S. N. Kulkov ${ }^{1,2,3, b)}$ \\ ${ }^{1}$ Institute of Strength Physics and Materials Science SB RAS, Akademicheskii Pr. 2/4, Tomsk, 634055 Russia \\ ${ }^{2}$ Tomsk Polytechnic University, Lenin Avenue 30, Tomsk, 634050 Russia \\ ${ }^{3}$ Tomsk State University, Lenin Avenue 36, Tomsk, 634050 Russia

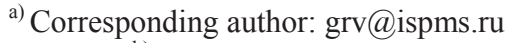 \\ b)kulkov@ispms.ru
}

\begin{abstract}
The microstructure of alumina ceramics based on powders with a varying grain size has been investigated. Both commercial alumina powders and those fabricated by denitration of aluminum salts in high-frequency discharge plasma were used. It is shown that the variation of the sintering temperature and morphology of the initial powders of the particles leads to a change of the pore structure of ceramics from pore isolated clusters to a structure consisting of a ceramic skeleton and a large pore space. Changing the type of pore structure occurs at about $50 \%$ of porosity. The ceramic pore size distribution is bimodal. Dependencies final density vs initial density are linear; at the same time with increasing temperature, inclination of changes from positive to negative, indicating the change of sealing mechanisms. Extrapolation of these curves showed that they intersect with the values of density of about $2 \mathrm{~g} / \mathrm{cm}^{3}$, which indicates the possibility of producing non-shrink ceramics. It is shown that the strength increases with increasing nanocrystalline alumina content in powder mixture. A change in the character the pore structure is accompanied by a sharp decrease in strength, which corresponds to the percolation transition in ceramics. These results showed that it is possible to obtain ceramic materials with the structure and properties similar to natural bone.
\end{abstract}

\section{INTRODUCTION}

Development of an integrated approach, which would allow you to create porous ceramic materials with predetermined structure and properties at various ratios of the micro-and nanocrystalline powders mixtures is an important task [1]. The use of nanocrystalline powders and mixtures with microcrystalline powders opens completely new opportunities to create biocompatible implants with different porosity for bone reconstruction.

A promising material for production of porous ceramic is alumina characterized by high strength, corrosion resistance, and resistance to attack by aggressive media [2]. While alumina-based ceramics offer obvious advantages over other materials, they are brittle, and their porous structure, as a rule, gives rise to a severe reduction in mechanical strength [3]. This is why alumina-based ceramics have a limited application. Nanosized ceramic powders in a non-equilibrium state open up new fields of use in production of high-porosity ceramics [4]. Due to a well-developed nanoparticle surface, special nanocrystalline structures with high bond strength along grain boundaries can be formed to provide high strength of the sintered ceramics [5].

A promising process for fabrication of high-porosity ceramics without using a standard practice based on burning out additives and pore agents is the mixing of coarse-grained and ultrafine-grained powders [6]. In this case, the pore geometry depends on the coarse-grained powder particle size and shape. However, in the porous ceramic materials produced in this way, ultrafine-grained powders serve merely as additives (as a rule, up to $10 \mathrm{wt}$. \%). While the data on the pore structure of the materials examined and on its association with the parameters of the fine crystal structure are of fundamental importance for an analysis of the deformation behavior of the ceramics under consideration, the mechanisms involved in the formation of the pore structure, using ultrafine-grained powders of varying contents, are poorly understood.

Physics of Cancer: Interdisciplinary Problems and Clinical Applications (PC'16) AIP Conf. Proc. 1760, 020022-1-020022-5; doi: 10.1063/1.4960241 Published by AIP Publishing. 978-0-7354-1418-1/\$30.00 
The objective of the present work is to study of the pore structure formation in sintered ceramic depending on the ratio of alumina powders with different particle size and determination of the main physical and mechanical properties.

\section{EXPERIMENT}

We have investigated two types of alumina powders: 99\% pure commercial alumina produced to All-Ruassian State Standard No. 30558-98 and plasma-chemical alumina powder fabricated by thermal decomposition of aqueous solution of aluminum nitrate in high-frequency discharge plasma (Specs. 2320-001-07622928-96). Detailed physical structure and mechanical properties of these powders was studied in [1].

Porous ceramics were prepared from powder mixtures with varying mixture ratios from commercial alumina to plasma-chemical $\mathrm{Al}_{2} \mathrm{O}_{3}$ powder. The powders were mixed in a rattler for $24 \mathrm{~h}$. Prior to pressing, 5 wt. \% polyvinyl alcohol was added to the mixture. Cylindrical samples were pressed in a hydraulic press at a pressure of $250 \mathrm{MPa}$. Sintering was performed at $1200,1300,1400,1500,1600$, and $1650^{\circ} \mathrm{C}$, the isothermal holding time was $1 \mathrm{~h}$.

An x-ray diffraction analysis of the ceramic samples was made in a diffractometer with filtered $\mathrm{CuK}_{\alpha}$ radiation. The average crystallite size (size of coherently diffracting domains) was calculated from small-angle reflection broadening. The level of crystal lattice microdistortion was calculated from distant-angle reflection broadening seen in the diffraction patterns. The pore structure morphology was studied by scanning electron microscopy (Quanta 200 3D). The pore size was calculated by the random intercept method. It took a minimum of 300 measurements. The data obtained were used to plot pore size distributions (bar charts), and the average pore size and standard deviation were calculated. Density was measured using the method of hydrostatic weighting, with the residual porosity derived from the density values. Shrinkage was calculated from geometric changes of the samples before and after sintering. An "Instron-1185" facility was used to determine the ultimate compressive strength.

\section{RESULTS AND DISCUSSION}

Since the plasma-chemical $\mathrm{Al}_{2} \mathrm{O}_{3}$ powder content in the samples was varied through a wide range and different sintering temperatures were used, a large set of states was produced in the ceramic materials under review. They were characterized by varying porosity, average crystallite size, and level of lattice microdistortions.

In the studied ceramics, bimodal distribution of pore sizes is observed (Fig. 1). The first maximum (d1) is due to pores less than $5 \mu \mathrm{m}$, the size of which is commensurate with the size of grains. A second maximum (d2) forms macropores ranging in size from 5 to $100 \mu \mathrm{m}$. Such large voids in ceramics are due to the formation of lasting frameworks made of used agglomerates powders during its pressing, the dimensions of which determine the size of macropores between them. In coarse fraction ceramic powder, the ratio of pore size of $0.5 \mu \mathrm{m}$ is substantially lower than the ceramic powders of the nanocrystalline $\mathrm{Al}_{2} \mathrm{O}_{3}$.

With the increase in the content of plasma chemical powder in the starting mixture the communicating pore space decrease and, thus, the reduction in the size of channeling is far. This is evidenced by reduction of the average pore size of from 53 to $9 \mu \mathrm{m}$ for plot $\mathrm{d} 2$ on Fig. 1. However, in ceramics that contain only plasmochemical powder the porosity is increasing again. In ceramics containing $90 \%$ plasma chemical powder, there is a structure with the highest density, and there are isolated pores with random shapes and pore separate clusters. Apparently, this is due to the fact that the alumina $10 \%$ additive added to the plasma chemical $\mathrm{Al}_{2} \mathrm{O}_{3}$ powder destroys the foam-like agglomerates plasma chemical powder on the pressing stage, wherein the particles and agglomerates of coarse alumina are not enough to form a lasting framework on their base.

We found that the increase in porosity is accompanied by a decrease in crystallite size and microstrain growth. Dependence of crystal lattice micro distortions porosity in alumina ceramics has a strongly pronounced break in the porosity of about $50 \%$.

Figure 2 depicts the dependence of the level of the crystal lattice microdistortions on the average grain size (size of the coherently diffracting domains) plotted with the use of the data for the samples with varying plasma-chemical powder content, sintering temperatures, and pore structure. The level of the lattice microdistortions is seen to decrease with increase in the grain size $D$.

The lattice microdistortions in coarse-grained materials revealed by the X-ray diffraction method are known to be largely due to point and linear lattice defects $[7,8]$. In addition, it is a matter of general experience that because of the well-developed system of grain boundaries, the lattice microdistortions in nanocrystalline materials may arise from the defect structure lying along grain boundaries [9]. 


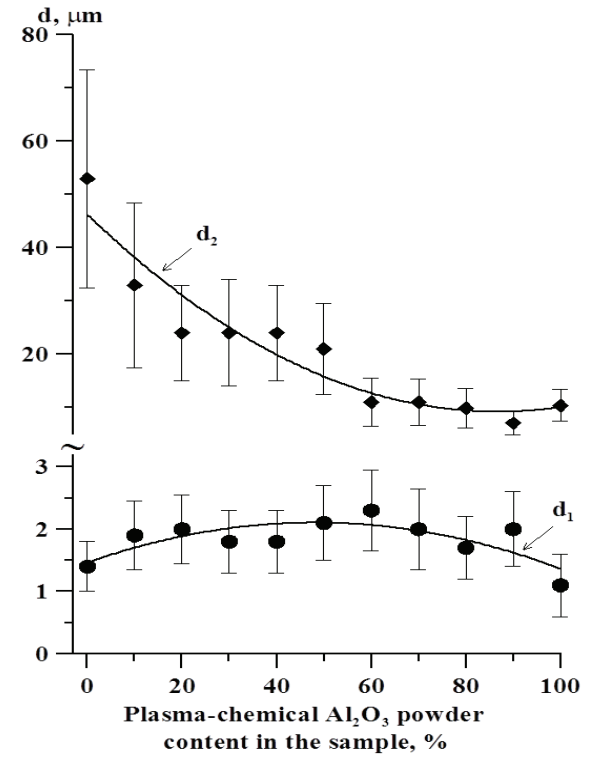

FIGURE 1. Dependence of an average pore size and standard deviation on the plasma-chemical $\mathrm{Al}_{2} \mathrm{O}_{3}$ powder content in the samples

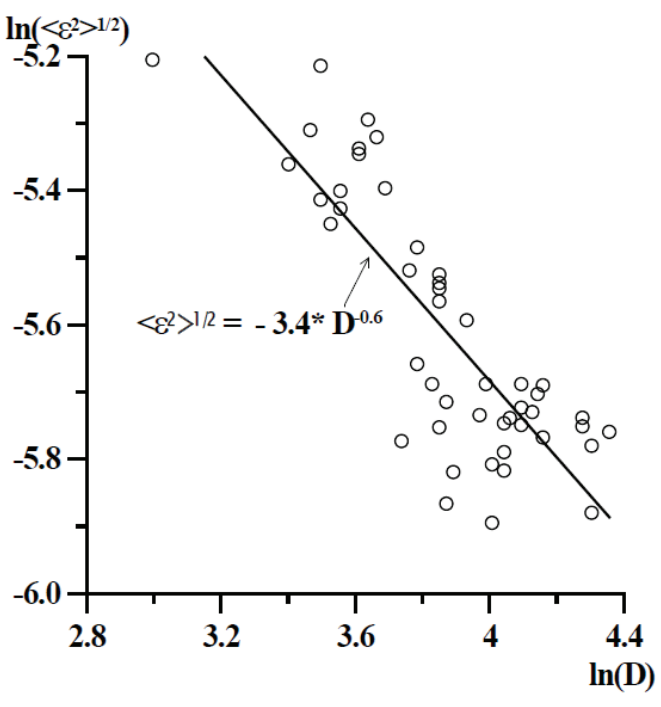

FIGURE 2. Dependence of the level of the crystal lattice microdistortions on the average grain size

The dependence shown in Fig. 4 is described by the following formula: $\left\langle\varepsilon^{2}\right\rangle^{1 / 2}=3.4 D^{-0.6}$. Here $D^{-0.6}$ corresponds to the case where all lattice microdistortions are due to the well-developed defect structure along grain boundaries [10].

The relationship was investigated between the fine crystal structure parameters and the sintering temperature, which indicates that, with an increase in the sintering temperature, the average crystallite size increases linearly, and the level of the crystal lattice microstrain decreases.

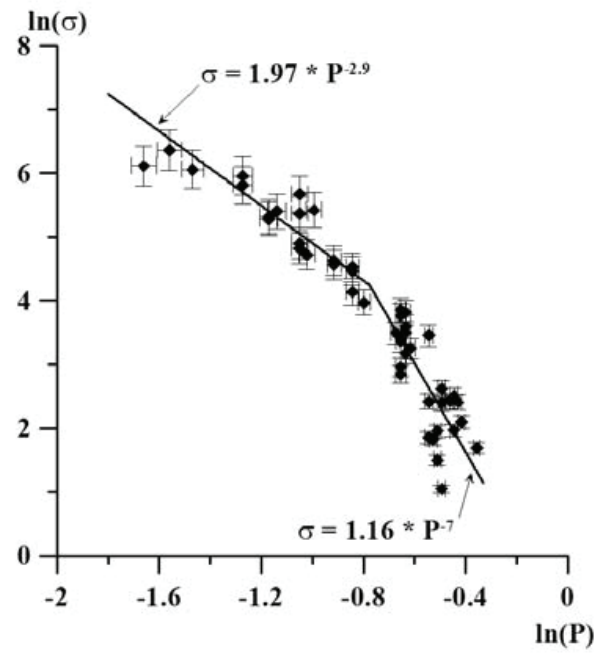

FIGURE 3. Compressive strength vs. porosity in the studied ceramics 


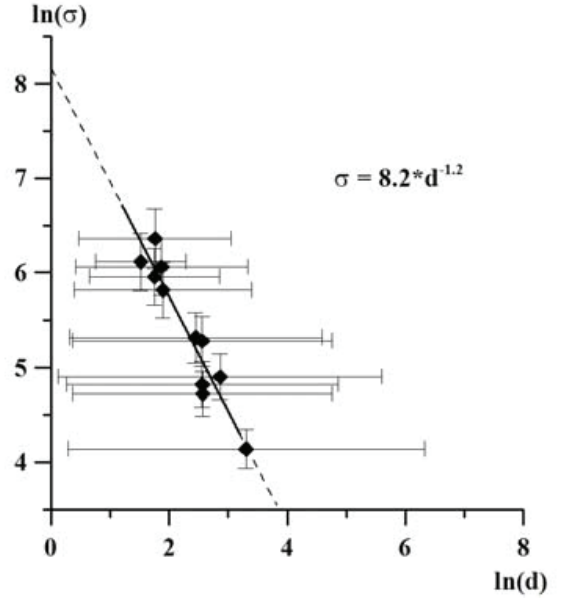

FIGURE 4. Compressive strength vs. average pore size in the studied ceramics

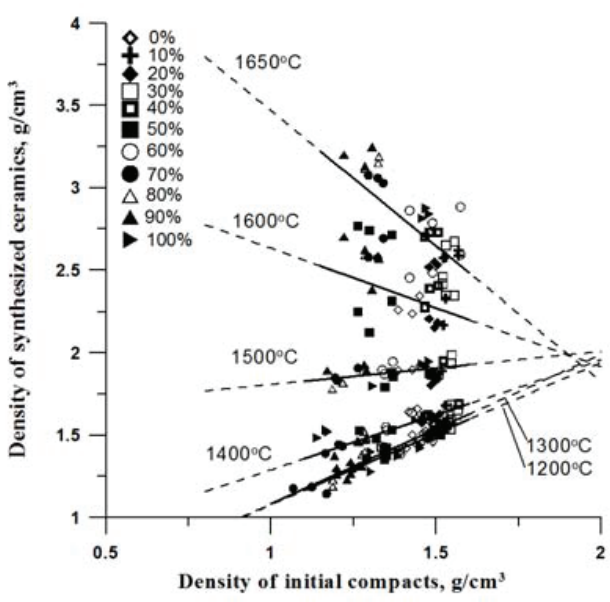

FIGURE 5. Density of ceramics compacts with different contents of plasma $\mathrm{Al}_{2} \mathrm{O}_{3}$ powder after sintering vs. initial density

Diffusion activation energy was estimated, which is equal to $97-103 \mathrm{~J} / \mathrm{mol}$ or $23 \mathrm{kcal} / \mathrm{mol}$, which is 5 times less than the activation energy of self-diffusion of aluminum and oxygen in polycrystalline oxide, from which we may suggest that the main mechanism of mass transfer during sintering is grain boundary diffusion.

Investigation of the effect of porosity on the strength of the ceramic showed that the strength of the samples decreases significantly with increasing porosity, when porosity is about $50 \%$, the angle of inclination of the approximating line is changed (Fig. 3). Primarily, it is due to the fact that there is a change in the nature from the isolated pores structure and pore clusters of various shapes and sizes for a ceramics structure consisting of two interpenetrating component-"substance-pore".

Thus, the changing of the pore structure nature is accompanied by a sharp decrease in the strength and growth of the crystal lattice microstrain, which corresponds to the percolation transition in system.

The pore volume and pore size also significantly affect the strength of the material. We found that the tensile strength is greater, the smaller the average pore size and dispersion (Fig. 4), and the extrapolation of this dependence to zero porosity showed that the strength of the studied ceramics will be about $3.5 \mathrm{GPa}$.

Dependences of the density of the synthesized ceramic from the initial density compacts, shown in Fig. 5, is linear. As can be seen from the graph, the density after sintering at temperatures of $1200-1400^{\circ} \mathrm{C}$ is higher and the higher initial density compacts while density after pressing greater in samples with a predominant content of coarse alumina. With further increase in the sintering temperature begins to play a major role not the initial density of the samples, and the number of plasma chemical $\mathrm{Al}_{2} \mathrm{O}_{3}$ powder in the initial mixture of powders, increase in the content of which results in changing the compression mechanisms and, as a consequence, increase in the density of the samples. This is reflected in the change of the angle of inclination of approximated straight at sintering temperatures above $1500^{\circ} \mathrm{C}$. After sintering at these temperatures, the porosity of the samples is about $50 \%$ and there is a change in the nature of the pore structure of the isolated for communicating, which corresponds to the percolation transition in ceramics under consideration.

Extrapolation of the straight lines in Fig. 5 (indicated by dotted lines) shows that they intersect in the region of $2 \mathrm{~g} / \mathrm{cm}^{3}$, indicating the possibility of a non-shrink ceramics.

\section{CONCLUSIONS}

It is shown that the variation of the sintering temperature, and morphology of the initial powders of the particles leads to a change in the pore structure of ceramics from pore isolated clusters to a structure consisting of a ceramic skeleton and a large pore space. Changing the type of pore structure occurs at about $50 \%$ porosity.

The ceramic pore size distribution is bimodal. The first peak is due to pores of size $2 \mu \mathrm{m}$, while the second peak decreases from 50 to $20 \mu \mathrm{m}$, with increasing content of $\mathrm{Al}_{2} \mathrm{O}_{3}$ samples nanocrystalline powder. 
Dependencies final density vs initial density are linear; at the same time with increasing temperature, inclination of changes from positive to negative, indicating the change of sealing mechanisms. Extrapolation of these curves showed that they intersect with the values of density of about $2 \mathrm{~g} / \mathrm{cm}^{3}$, which indicates the possibility of producing non-shrink ceramics.

It is shown that the strength increases with increasing nanocrystalline alumina content in powder mixture. Change in the character the pore structure is accompanied by a sharp decrease in strength, which corresponds to the percolation transition in ceramics.

These results had shown that one can possible to obtain ceramic materials with structure and properties similar to natural bone.

\section{ACKNOWLEDGMENTS}

The research was conducted in framework Siberian Branch Program, project No. III.23.2.3.

The study reported in this article was conducted according to accepted ethical guidelines involving research in humans and/or animals and was approved by an appropriate institution or national research organization.

The study is compliant with the ethical standards as currently outlined in the Declaration of Helsinki.

All individual participants discussed in this study, or for whom any identifying information or image has been presented, have freely given their informed written consent for such information and/or image to be included in the published article.

\section{REFERENCES}

1. M. V. Grigoriev and S. N. Kulkov, Ěpitöanyag 3, 66-69 (2010).

2. V. V. Guliants, M. A.Carreon, and Y. S. Lin, J. Membr. Sci. 235, 53-72 (2004).

3. F. Tang, H. Fudoiizi, and Y. Sakka, J. Am. Ceram. Soc. 86, 2050-2054 (2003).

4. C. Kaya, J. Y. He, X. Gu, and E. G. Butler, Microporous Mesoporous Mater. 54, 37 (2002).

5. M. A. Meyers, A. Mishra, and D. J. Benson, Progr. Mat. Sci. 51, 427-556 (2006).

6. M. V. Grigoriev and S. N. Kulkov, Rus. Phys. J. 12, 1305-1311 (2011).

7. Ya. O. Umanskii, Yu. A. Skakov, and A. N. Ivanov, Crystallography, Roentgenography and Electron Microscopy (Metallurgiya, Moscow, 1982), pp. 86-95.

8. A. Guinier, Theorieet technique de la radiocristallographie (Dunod, Paris, 1956), pp. 15-48.

9. A. I. Gusev, Phys. Usp. 41, 49-76 (1998).

10. P. V. Korolev and S. N. Kulkov, Perspektiv. Mater. 2, 55-61 (1998). 